\title{
Effects of ROI Placement on PET-Based Assessment of Tumor Response to Therapy
}

\author{
Mike Sattarivand, ${ }^{1,2}$ Curtis Caldwell, ${ }^{1,2,3}$ Ian Poon, ${ }^{4,5}$ Hany Soliman, ${ }^{5}$ and Katherine Mah ${ }^{2,4}$ \\ ${ }^{1}$ Department of Medical Biophysics, University of Toronto, Ontario Cancer Institute, Princess Margaret Hospital, \\ 610 University Avenue, Room 7-411, Toronto, ON, Canada M5G 2M9 \\ ${ }^{2}$ Department of Medical Physics, Odette Cancer Centre, Sunnybrook Health Sciences Center, 2075 Bayview Avenue, \\ Room TG-217, Toronto, ON, Canada M4N 3M5 \\ ${ }^{3}$ Department of Medical Imaging, Faculty of Medicine, University of Toronto, 263 McCaul Street, 4th Floor, \\ Toronto, ON, Canada M5T 1W7 \\ ${ }^{4}$ Department of Radiation Oncology, University of Toronto, FitzGerald Building, 150 College Street, Room 106, \\ Toronto, ON, Canada M5S $3 S 2$ \\ ${ }^{5}$ Department of Radiation Oncology, Odette Cancer Centre, Sunnybrook Health Sciences Center, Toronto, ON, Canada M4N 3 M5
}

Correspondence should be addressed to Mike Sattarivand; mike.sattarivand@sunnybrook.ca

Received 4 September 2012; Revised 15 January 2013; Accepted 7 February 2013

Academic Editor: Adriaan A. Lammertsma

Copyright (C) 2013 Mike Sattarivand et al. This is an open access article distributed under the Creative Commons Attribution License, which permits unrestricted use, distribution, and reproduction in any medium, provided the original work is properly cited.

Purpose. Quantitative PET response assessment during therapy requires regions of interest (ROI). Commonly, a fixed-size ROI is placed at the maximum uptake point in the pretreatment study. For intratreatment, the ROI is placed either at the maximum uptake point $\left(\mathrm{ROI}_{\text {peak }}\right)$ or at the same location as the pretreatment $\mathrm{ROI}\left(\mathrm{ROI}_{\text {same }}\right)$. We have evaluated the effects of the ROI placement on response assessment. Methods. PET scans of 15 head and neck cancer patients were used to evaluate the effects of the two ROI methods on response assessment. Results. The average intratreatment $\mathrm{ROI}_{\text {peak }}$ uptake was $13.4 \%$ higher than the ROI $\mathrm{Iame}_{\text {uptake }}$ (range $-14 \%$ to $38 \%$ ). The average relative change in $\mathrm{ROI}_{\text {peak }}$ uptake was $7.9 \%$ lower than $\mathrm{ROI}_{\text {same }}$ uptake (range $-5 \%$ to $36 \%$ ), resulting in ambiguous tumour classification in 19\% of the tumours. Conclusion. Quantitative PET response assessment using a fixed-size ROI is sensitive the ROI placement. The difference between $\mathrm{ROI}_{\text {peak }}$ and $\mathrm{ROI}_{\text {same }}$ could be substantial resulting in ambiguous response assessment. Although the fixed-size ROI is simple to implement, it is also prone to the limitations and should be used with caution. Clinical trial data are necessary to establish reliable thresholds for fixed-size ROI techniques and to evaluate their efficacy for response assessment.

\section{Introduction}

As a powerful molecular imaging tool, positron emission tomography (PET) is increasingly being used for early assessment of tumour response to therapy [13-15]. Typically two sequential PET studies are performed and the tumor standardized uptake value (SUV) in the pre-treatment (Pre-Tx) study is compared to that of the intra-treatment (Intra-Tx) study.

Response assessment using SUVs requires the selection of either a representative tumor voxel or a region of interest
(ROI) for quantification. One of the simplest and most common methods of quantifying tumor uptake is to use the single voxel containing the maximum SUV $\left(\operatorname{SUV}_{\max }\right)[16,17]$. Unfortunately, $\mathrm{SUV}_{\max }$ values are highly sensitive to image noise and voxel size $[18,19]$, which leads to uncertainties in quantitative response assessment. Moreover, Krak, et al. [19] reported that $S U V_{\text {max }}$ has poor reproducibility compared to estimates of SUV made using ROI methods. As a more robust alternative, an average SUV within a small fixed size ROI has been recommended to provide adequate statistical 
TABLE 1: A summary of previous Intra-Tx tumour response assessment studies that used the Fixed size ROI method.

\begin{tabular}{|c|c|c|c|c|c|}
\hline Study & $n$ & Site & Pre-Tx ROI & Intra-Tx ROI & Res. Thr \\
\hline $\begin{array}{l}\text { Schelling et al. } \\
(2000)[1]\end{array}$ & 22 & Breast & Fixed size, circular $15 \mathrm{~mm}$ at $\max$ & Fixed size, circular $15 \mathrm{~mm}$ at $\max$ & $55 \%$ \\
\hline $\begin{array}{l}\text { Weber et al. (2003) } \\
{[2]}\end{array}$ & 57 & Lung & Fixed size, circular $15 \mathrm{~mm}$ at $\max$ & Fixed size, circular $15 \mathrm{~mm}$ at $\max$ & $20 \%$ \\
\hline $\begin{array}{l}\text { Avril et al. (2005) } \\
{[3]}\end{array}$ & 33 & Ovarian & Fixed size, circular $15 \mathrm{~mm}$ at $\max$ & Fixed size, circular $15 \mathrm{~mm}$ at $\max$ & $20 \%$ \\
\hline $\begin{array}{l}\text { Brun et al. (2002) } \\
{[4]}\end{array}$ & 47 & Head and neck & Fixed size, square ( 4 or 9 pixels) at $\max$ & Fixed size, square ( 4 or 9 pixels) at max & Median \\
\hline $\begin{array}{l}\text { Rousseau et al. } \\
\text { (2006) [5] }\end{array}$ & 64 & Breast & Fixed size, 5 to $10 \mathrm{~mm}$ at $\max$ & Fixed size, 5 to $10 \mathrm{~mm}$ at $\max$ & $40 \%$ \\
\hline $\begin{array}{l}\text { Maisonobe et al. } \\
\text { (2013) [6] }\end{array}$ & 40 & Colorectal & Fixed size, $3 \times 3 \times 3$ voxels at $\max$ & Fixed size, $3 \times 3 \times 3$ voxels at $\max$ & $\mathrm{N} / \mathrm{Sp}$ \\
\hline Ott et al. (2006) [7] & & EG junction & Fixed size, circular $15 \mathrm{~mm}$ at $\max$ & $\begin{array}{l}\text { Fixed size, circular } 15 \mathrm{~mm} \text { at the same } \\
\text { position using landmark }\end{array}$ & $35 \%$ \\
\hline $\begin{array}{l}\text { Weber et al. (2001) } \\
\text { [8] }\end{array}$ & 40 & EG junction & Fixed size, circular $15 \mathrm{~mm}$ at $\max$ & $\begin{array}{l}\text { Fixed size, circular } 15 \mathrm{~mm} \text { at the same } \\
\text { position using landmark }\end{array}$ & $35 \%$ \\
\hline $\begin{array}{l}\text { Wieder et al. } \\
\text { (2007) [9] }\end{array}$ & 24 & EG junction & Fixed size, circular $15 \mathrm{~mm}$ at $\max$ & $\begin{array}{l}\text { Fixed size, circular } 15 \mathrm{~mm} \text { at the same } \\
\text { position using landmark }\end{array}$ & $35 \%$ \\
\hline $\begin{array}{l}\text { Ott et al. (2003) } \\
{[10]}\end{array}$ & 44 & Gastric & Fixed size, circular $15 \mathrm{~mm}$ at $\max$ & $\begin{array}{l}\text { Fixed size, circular } 15 \mathrm{~mm} \text { at the same } \\
\text { position using landmark }\end{array}$ & $35 \%$ \\
\hline $\begin{array}{l}\text { Wieder et al. } \\
\text { (2004) [11] }\end{array}$ & 38 & Esophagus & Fixed size, circular $15 \mathrm{~mm}$ at $\max$ & $\mathrm{N} / \mathrm{Sp}$ & $30 \%$ \\
\hline $\begin{array}{l}\text { Schwarz et al. } \\
\text { (2005) [12] }\end{array}$ & 11 & Breast & $\begin{array}{l}\text { Fixed size, circular (size N/Sp) } \\
\text { manually placed }\end{array}$ & $\begin{array}{l}\text { Fixed size, circular (size N/Sp) manually } \\
\text { placed }\end{array}$ & $20 \%$ \\
\hline
\end{tabular}

$n$ : number of patients, Pre-Tx: pretreatment, Intra-Tx: intratreatment, ROI: region of interest, Res. Thr.: response threshold, EG: esophagogastric, N/Sp: not specified.

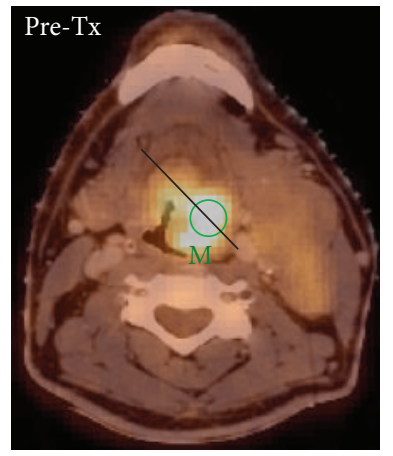

(a)

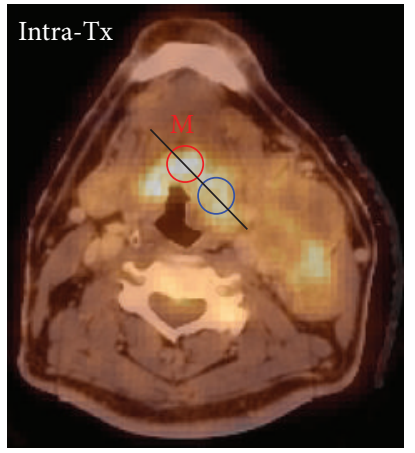

(b)

FIGURE 1: Change in the distribution of FDG uptake during treatment. The PET/CT images of Pre-Tx (a) and Intra-Tx (b) are of a patient with a base of tongue primary tumour. Two circular ROIs of $15 \mathrm{~mm}$ diameter are centered at the maximum uptake points on both Pre-Tx (green) and Intra-Tx (red) images denoted by "M." An additional $15 \mathrm{~mm}$ diameter circular ROI is placed on the Intra-TX image (blue) in a position judged to correspond to the same anatomical location as the ROI as in the Pre-Tx. The FDG uptake profiles along the black lines connecting the two Intra-Tx ROIs are shown in Figure 2.

quality in SUV measurements and to reduce uncertainties in quantitative response assessment [16].

Table 1 lists representative studies [1-12] that have used the fixed-size ROI method for early tumour response assessment. The Pre-Tx ROI is usually centred on the SUV max $_{\text {voxel. }}$ However, there are two distinct approaches to the placement of the Intra-Tx ROI. Some studies have centred the IntraTx ROI on the SUV $\mathrm{max}_{\text {voxel }}$ (ROI $\mathrm{I}_{\text {peak }}$ ), whereas others have placed it at the same location as it was in the Pre-Tx image using anatomical landmarks $\left(\mathrm{ROI}_{\text {same }}\right)$.
The distribution of uptake within the tumour may change in response to therapy such that the maximum uptake point in the Intra-Tx study is found at an anatomically different location than it was prior to treatment. This is illustrated in Figures 1 and 2 for a sample head and neck cancer (HNC) patient. Figure 2 illustrates two quantitative response assessments based on the two different choices of Intra-Tx ROI placement.

Using the $\mathrm{ROI}_{\text {same }}$ method is reasonable if the goal is to evaluate the change in uptake in the same area of the 


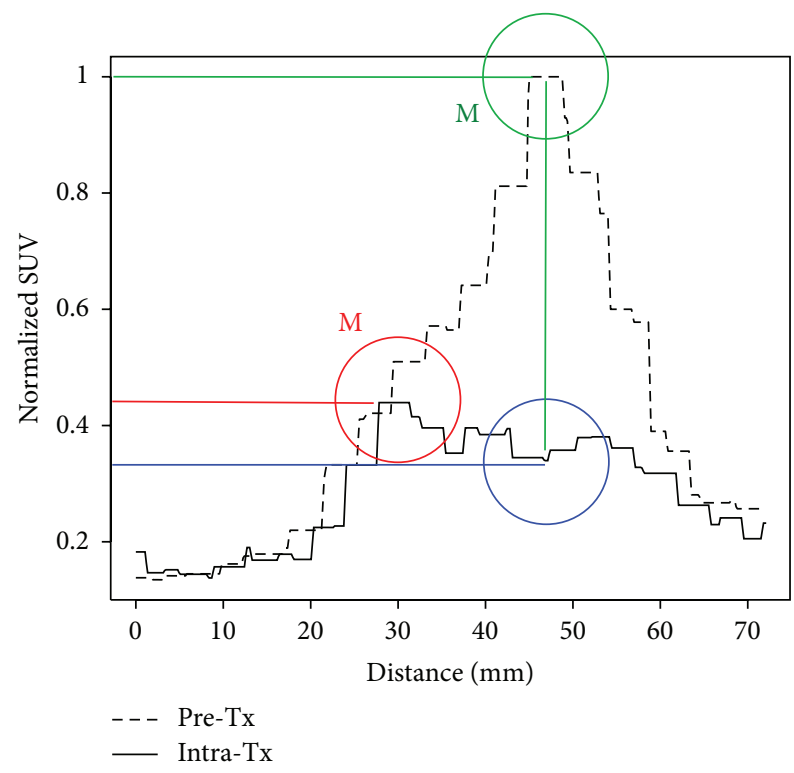

FIgUre 2: The uptake profile from Figure 1 normalized to Pre-Tx maximum SUV. The distribution of uptake within the tumour has changed during the therapy such that the maximum uptake point along the profile in Pre-Tx corresponds to a local minimum uptake point in the Intra-Tx. The maximum uptake point along the profile in Intra-Tx is now in a different location of the tumour.

tumour. This method has been recommended by the European Organization for Research and Treatment of Cancer (EORTC) [20]. However, unlike $\mathrm{ROI}_{\text {peak }}$, tumour response measured by $\mathrm{ROI}_{\text {same }}$ is prone to uncertainty due to the difficulty in positioning an ROI in the Intra-Tx scan in the exact anatomic location as it was in the Pre-Tx scan. Geometric changes of both tumour and normal tissues may occur during the therapy making it difficult to place an ROI at exactly the same location as it was in the Pre-Tx scan using anatomical landmarks. Figure 3 shows PET/CT images of a sample HNC patient illustrating the magnitude of typical geometric changes in terms of volume losses and shifts.

Uncertainty in the placement of the Intra-Tx ROI could significantly affect the accuracy of quantitative response assessment. Uncertainties in quantitative response assessment could have significant impact on treatment decisions and clinical outcome. Consequently, we investigated the effects of fixed-size ROI placement on quantitative response assessment. The purpose of this study was twofold: (1) to evaluate quantitative response assessment when Intra-Tx PET images are measured using the $\mathrm{ROI}_{\text {peak }}$ and $\mathrm{ROI}_{\text {same }}$ methods; (2) to quantify the geometric changes of both tumour and normal tissues and their impact on quantitative response assessment using the $\mathrm{ROI}_{\text {same }}$ method.

\section{Materials and Methods}

2.1. Design of the Study. Two independent populations (A and B) were used. Population A consisted of 15 patients with a total of 38 gross tumour volumes (GTV) identified by experienced radiation oncologists. Population A was used to compare two quantitative tumour response assessments based on using the $\mathrm{ROI}_{\text {peak }}$ and $\mathrm{ROI}_{\text {same }}$ methods. Population $B$ consisted of 10 patients with a total of 33 GTVs identified by experienced radiation oncologists and was used to quantify geometric changes of both tumour and normal tissues during therapy. The impact of these geometric changes on quantitative tumour response assessment was evaluated in population A. Both populations A and B were part of a clinical trial at Sunnybrook Health Sciences Centre (Toronto, Canada) to assess tumour response in patients with advanced HNC. Population B consisted of patients entered in the pilot study which proceeded the main trial while population A consisted of patients entered in the clinical trial itself.

While populations A and B were very similar, there were some slight differences, primarily in the CT-voxel size used and the average time between the Pre-Tx and IntraTx scans. All patients in both groups had locally advanced HNC (stage III or IV) and underwent 6.6 weeks of radical radiotherapy with concurrent chemotherapy. Patients received intensity modulated radiation therapy (IMRT) of 70 Gy in 33 fractions to all GTVs for both primary (GTVp) and involved lymph nodes (GTVn). All patients also received concurrent bolus platinum chemotherapy as tolerated by intravenously injecting $100 \mathrm{mg} / \mathrm{m}^{2}$ Cisplatin on days 1,22 , and 43. Patients underwent two sequential FDG-PET/CT scans, one Pre-Tx and one Intra-Tx, both supine in the same position using a thermoplastic radiotherapy immobilization mask. One $18 \mathrm{~cm}$ axial field of view (FOV) that covered the head and neck area was used. The PET/CT scanner was the GEMINI System (Philips Medical System, Cleveland, Ohio). Prior to the PET/CT scans, patients were injected with $5 \mathrm{MBq}$ of FDG per $\mathrm{kg}$. Patients heavier than $75 \mathrm{~kg}$ were injected with a fixed dose of $370 \mathrm{MBq}$ of FDG. PET images were reconstructed using a 3-Dimensional RowAction Maximum Likelihood Algorithm (3D-RAMLA) and corrected for attenuation using CT. In order to register the Intra-Tx CT to the Pre-Tx CT images, a Chamfer matching algorithm [21] based on bony structures was implemented in house using the Interactive Data Language (IDL) Ver. 6.4 (Research Systems Inc., Boulder, CO). The algorithm used 3D rigid body with rotation and translation but no scaling. An IDL program was also developed in house to simultaneously display the registered Pre-Tx and Intra-Tx PET/CT images, to contour ROIs, and to read SUV values. PET images were interpolated to match the voxel sizes of CT images. All statistical analyses were performed using the public domain package "R" (http://www.r-project.org/).

2.2. Population A. Pre-Tx FDG PET/CT scans were performed $14 \pm 4$ days (range, $8-22$ ) prior to the start of the treatment. Intra-Tx FDG PET/CT scans were performed 16 \pm 2 days (range, 11-20) after the first treatment day. The CT-voxel size was $0.59 \times 0.59 \times 1.60 \mathrm{~mm}^{3}$ and the CT FOV was $300 \times 300 \times 210 \mathrm{~mm}^{3}$ in lateral, anterior-posterior, and superior-inferior directions, respectively. The PET voxel size was $2 \times 2 \times 2 \mathrm{~mm}^{3}$ and the PET FOV was $576 \times$ $576 \times 180 \mathrm{~mm}^{3}$ in lateral, anterior-posterior, and superiorinferior directions, respectively. PET images were acquired 


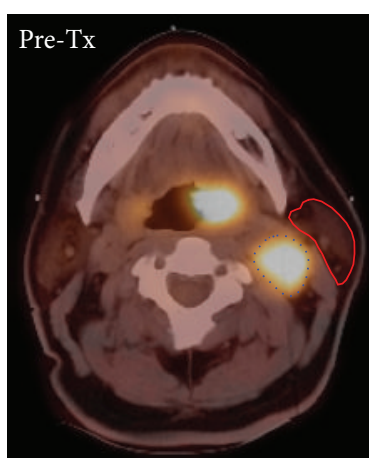

(a)

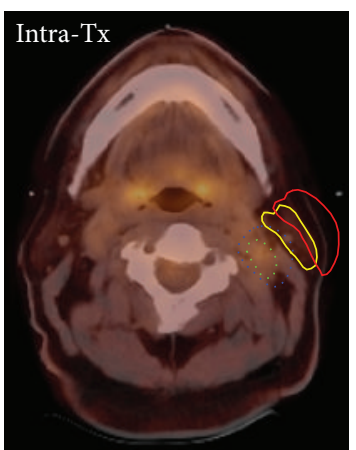

(b)

Figure 3: Both tumors and normal tissues may shrink and shift during the treatment. The coregistered PET/CT images of Pre-Tx (a) and Intra-Tx (b) are cross-sectional images of a patient with a primary tumour of the tonsil. The patient's left parotid gland in Intra-Tx (yellow contour) shows volume loss and shift relative to that in Pre-Tx (red contour). Similarly, the gross tumour volume for one nodal disease site in Intra-Tx (dotted green contour) shows volume loss and shift relative to that in Pre-Tx tumour (dotted blue contour).

50 minutes after injection for 2.5 minutes. The Pre-Tx and Intra-Tx PET postinjection acquisition times were matched within 5 minutes.

The SUVs were normalized to the patients' body weight. For each GTV, ROI peak (a circular ROI of $15 \mathrm{~mm}$ diameter) was placed on a single transaxial slice centered at the maximum FDG uptake point in both Pre-Tx and Intra-Tx images. For each GTV, ROI same (a circular ROI of $15 \mathrm{~mm}$ diameter) was also placed on a single transaxial slice at the location of the Intra-Tx image that corresponded to the same physical location as the Pre-Tx max-point ROI. A dual-board certified, nuclear medicine/radiology physician positioned $\mathrm{ROI}_{\text {same }}$ based on anatomical landmarks. Thus, each GTV had two Intra-Tx ROIs. The distance between the centers of these two ROIs was measured in 3D geometry.

On the same transaxial slice where $\mathrm{ROI}_{\text {same }}$ was located, the Intra-Tx GTV size was measured by averaging the anterior-posterior and lateral extents of an oncologist drawn GTV. In order to reduce errors in FDG uptake from partial volume effects, only Intra-Tx GTVs larger than $15 \mathrm{~mm}$ were subsequently analyzed, reducing the total number of GTVs available for analysis from 38 to 26 .

Tumour response assessments were obtained using two different methods, called $\Delta \mathrm{SUV}_{\text {peak }}$ and $\Delta \mathrm{SUV}_{\text {same }}$, by calculating the relative change in tumour uptake:

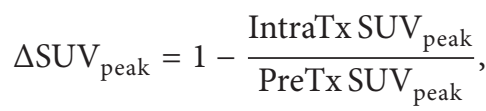

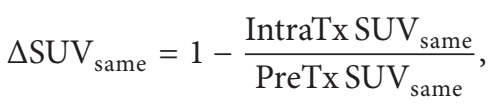

where $\mathrm{SUV}_{\text {peak }}$ is the mean SUV within $\mathrm{ROI}_{\text {peak }}$ in either Pre-

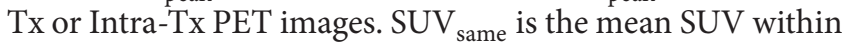
the $\mathrm{ROI}_{\text {same }}$ in the Intra-Tx PET image. A positive value for $\Delta \mathrm{SUV}_{\text {peak }}$ or $\Delta \mathrm{SUV}_{\text {same }}$ indicates a decrease in uptake and a negative value indicates an increase in uptake.

In order to determine how uncertainties in positioning $\mathrm{ROI}_{\text {same }}$ due to geometric changes may impact $\triangle \mathrm{SUV}_{\text {same }}$ values, the original $\mathrm{ROI}_{\text {same }}$ was systematically shifted in a $3 \mathrm{D}$ grid geometry up to $25 \mathrm{~mm}$ in three orthogonal directions. The sampling spaces of the grid were $1.17,1.17$, and $1.60 \mathrm{~mm}$ in the lateral, anterior-posterior, and superior-inferior directions, respectively. For each point in the grid $\mathrm{SUV}_{\text {same }}$ was determined. This data set was sorted based on the distance

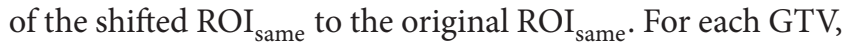
$\mathrm{SUV}_{\text {same }}$ was calculated and plotted as a function of this distance (i.e., positioning error). Each plot was normalized to the

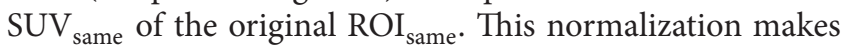
the $y$-axes represented also normalized $\left(1-\Delta \mathrm{SUV}_{\text {same }}\right)$. Plots of normalized SUV $\mathrm{Same}_{\text {same }}$ were averaged over $16 \mathrm{GTV}$ with Intra-Tx size smaller than $30 \mathrm{~mm}$ or $10 \mathrm{GTVs}$ with IntraTx size larger than $30 \mathrm{~mm}$. The arbitrary $30 \mathrm{~mm}$ threshold (twice the ROI size) was chosen to emphasize the effects due primarily to tumour uptake heterogeneity versus the effects due primarily to the partial volume effect. Tumour uptake heterogeneity was expected to have greater impact in large GTVs $(>30 \mathrm{~mm})$ and the partial volume effect was expected to have greater impact in small GTVs $(<30 \mathrm{~mm})$.

2.3. Population B. Pre-Tx FDG PET/CT scans were performed $17 \pm 5$ days (range, 13-28) prior to the start of the treatment. Intra-Tx FDG PET/CT scans were performed 33 \pm 4 days (range, 28-40) after the first treatment day. The CT-voxel size was $1.17 \times 1.17 \times 6.5 \mathrm{~mm}^{3}$ and the CT FOV was $600 \times 600 \times 208 \mathrm{~mm}^{3}$ in the lateral, anterior-posterior, and superior-inferior directions, respectively. The PET voxel size was $2 \times 2 \times 2 \mathrm{~mm}^{3}$ and the PET FOV was $576 \times$ $576 \times 180 \mathrm{~mm}^{3}$ in the lateral, anterior-posterior, and superiorinferior directions, respectively.

GTVs were contoured manually by an oncologist experienced in treatment of HNC. All the GTVs were contoured on CT images guided by coregistered PET images. Noncoregistered diagnostic MR images were available to aid contouring in all patients except one where no MRI study was performed. Radiology reports on both PET/CT and MRI studies were also used to aid in contouring.

Geometric changes of the GTVs and normal tissues during treatment were thought to be potentially important 


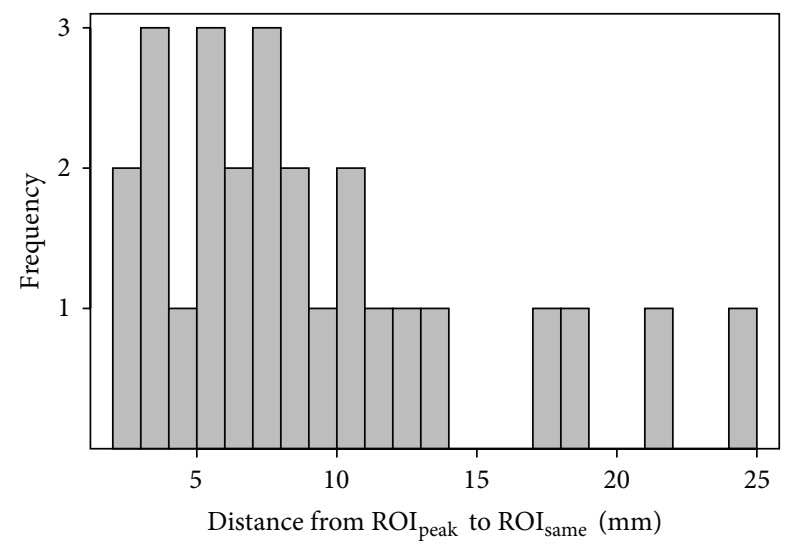

FIGURE 4: Histogram of distances between the centers of the two Intra-Tx ROIs.

in influencing the accuracy of placement of an ROI for quantitative tumour response assessment. In addition to GTVs, geometric changes of some normal tissues were also quantified. While the geometric shifts in tumours, not normal tissues, were of primary interest, the uncertainty in estimating geometric shifts in GTVs was greater than the uncertainty in estimating shifts in other structures, simply due to the difficulty in accurately delineating the GTV after treatment. Thus, the geometric shifts in normal tissues were used as surrogate measures of possible shifts in GTVs. Ten normal tissues were contoured on both Pre-Tx and Intra-Tx CT images for each patient. These normal tissues included the $\mathrm{C} 2$ vertebral body, mandible, hyoid, spinal cord, right and left sternocleidomastoid muscles, right and left parotid glands, and right and left submandibular glands. All normal tissues were contoured using consistent window and level settings under the guidance of an experienced oncologist. The most inferior extent for contouring the spinal cord and the sternocleidomastoid muscles was the most superior aspect of the apex of the lung. The most superior extent of the spinal cord was chosen to correspond to the most superior extent of the $\mathrm{C} 2$ vertebral body. Mandible and parotid contours were excluded from one patient since the scan did not include the entire organs in the superior direction.

Using both Pre-Tx and Intra-Tx contours for normal tissues and GTVs, an IDL program was developed in house to quantify the geometric changes by calculating

(1) percentage volume changes, that is, Intra-Tx volume relative to Pre-Tx volume,

(2) shift of center Of mass (COM), that is, Intra-Tx COM relative to Pre-Tx COM. The shifts were calculated as a shift vector in a $3 \mathrm{D}$ geometry and the reported values are the absolute values of these vectors.

\section{Results}

Patient characteristics for both populations are listed in Table 2.
TABLE 2: Patient characteristics.

\begin{tabular}{lcc}
\hline & Population A & Population B \\
\hline No. of patients & 15 & 10 \\
Sex $(\mathrm{F}, \mathrm{M})$ & $3 \mathrm{~F}, 12 \mathrm{M}$ & $1 \mathrm{~F}, 9 \mathrm{M}$ \\
Age & & \\
$\quad$ Mean age \pm SD & $58.3 \pm 5.7 \mathrm{yr}$ & $58.7 \pm 11.6 \mathrm{yr}$ \\
$\quad$ Age range & $49-68 \mathrm{yr}$ & $42-79 \mathrm{yr}$ \\
Clinical stage & 5 & \\
$\quad$ Stage III & 10 & 1 \\
$\quad$ Stage IV & 38 & 9 \\
Total no. of GTV & 15 & 10 \\
$\quad$ GTVp & 23 & 23 \\
$\quad$ GTVn & & \\
Site & 5 & 4 \\
Tongue & 3 & 1 \\
Tonsil & 5 & 1 \\
$\quad$ Hypopharynx & 2 & 1 \\
Larynx & 0 &
\end{tabular}

F: female, M: male, SD: standard deviation, GTV: gross tumour volume, GTVp: primary tumour, GTVn: involved lymph node.

3.1. Population $A$. The mean Intra-Tx GTV size (i.e., average of the anterior-posterior and lateral extents) was $25.7 \pm$ $8.9 \mathrm{~mm}$ (range, 15.1-46.5). A histogram of the distances between the centers of the two Intra-Tx ROIs for each GTV is shown in Figure 4. This histogram shows that the IntraTx maximum uptake point does not normally correspond to the same physical location as that for Pre-Tx. The median distance between the centers of the two Intra-Tx ROIs was $7.4 \mathrm{~mm}$. The two Intra-Tx ROIs were on the same transaxial slice in only $8 \%$ of the cases (in 2 out of 26 GTVs).

Figure 5(a) shows a scatter plot comparing quantitative tumour response assessments using the $\mathrm{ROI}_{\text {peak }}$ and $\mathrm{ROI}_{\text {same }}$ approaches. A high two-sided Pearson correlation coefficient was found between $\Delta \mathrm{SUV}_{\text {peak }}$ and $\Delta \mathrm{SUV}_{\text {same }}(r=0.93, p=$ $7 e-12)$ for all GTVs. Similarly, the $r$ value between Intra-Tx $\mathrm{SUV}_{\text {peak }}$ and $\Delta \mathrm{SUV}_{\text {same }}$ was $0.92, p=5 e-11$ for all GTVs.

As expected, Intra-Tx SUV $\mathrm{Veak}_{\text {pad }}$ hagher value than $\mathrm{SUV}_{\text {same }}$ for most GTVs, resulting in a lower value for $\Delta \mathrm{SUV}_{\text {peak }}$ compared to $\Delta \mathrm{SUV}_{\text {same }}$ as seen in Figure 5(a). On average, $\mathrm{SUV}_{\text {peak }}$ was $13.4 \%$ higher than $\mathrm{SUV}_{\text {same }}$ (range - $14 \%$ to $38 \%$ ) and $\Delta \mathrm{SUV}_{\text {peak }}$ was $7.9 \%$ lower than $\Delta \mathrm{SUV}_{\text {same }}$ (range $-5 \%$ to $36 \%)$. One unusual case, identified by the oblique arrow in Figure 5(a), is an example where the $\Delta \mathrm{SUV}_{\text {peak }}$ was $5.3 \%$ higher than $\Delta \mathrm{SUV}_{\text {same. }}$. In this case, the ROI $\mathrm{R}_{\text {peak }}$ region placed centred on the peak voxel in the Intra-Tx scan actually had a lower average uptake than the $\mathrm{ROI}_{\text {same }}$ region. GTVs in Figure 5 are coded for primary versus nodal mass as well as for large $(>30 \mathrm{~mm})$ versus small $(<30 \mathrm{~mm})$ GTVs. No statistically significance difference was found between $\Delta S U_{\text {peak }}$ and $\Delta S \mathrm{SU}_{\text {same }}$ on the basis of GTV size (large versus small) or type (primary versus node).

Figure 5(b) shows classification of individual tumours based on the PET Response Criteria in Solid Tumors (PERCIST) [16] using either $\Delta \mathrm{SUV}_{\text {peak }}$ or $\Delta \mathrm{SUV}_{\text {same }}$. 


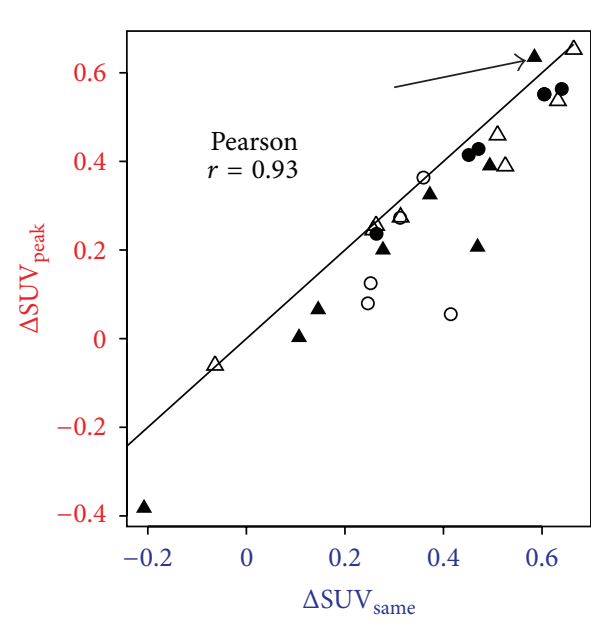

- Primary tumor $>30 \mathrm{~mm}$

- Primary tumor $<30 \mathrm{~mm}$

- Positive node $>30 \mathrm{~mm}$

$\triangle$ Positive node $<30 \mathrm{~mm}$

(a)

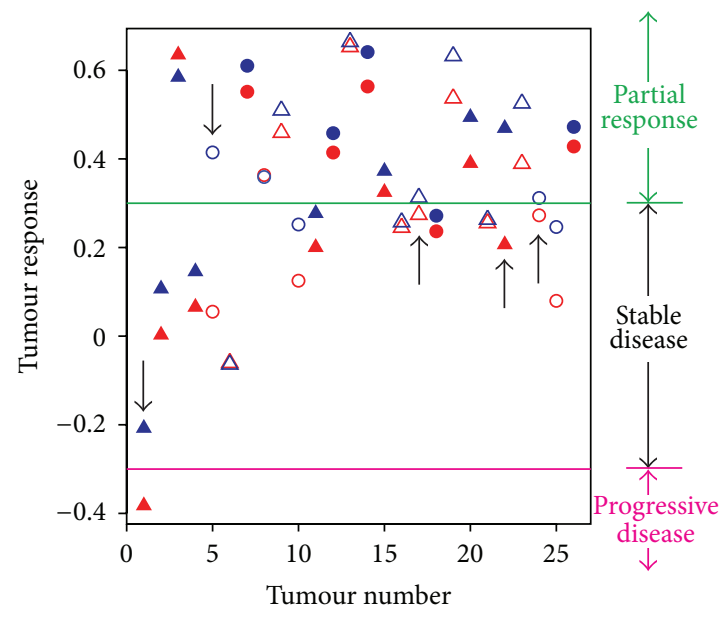

- Primary tumor $>30 \mathrm{~mm}$

- Primary tumor $<30 \mathrm{~mm}$

- Positive node $>30 \mathrm{~mm}$

$\Delta$ Positive node $<30 \mathrm{~mm}$

(b)

FIGURE 5: Comparison between the two quantitative tumour response measurements when two different Intra $=$ Tx ROI methods were used. Plot (a) is a scatter plot of the two methods. The solid line in this graph is the unity line where $\Delta \mathrm{SUV}_{\text {peak }}=\Delta \mathrm{SUV}$ same . For most tumours $\Delta \mathrm{SUV}_{\text {peak }} \leq \Delta \mathrm{SUV}_{\text {same. }}$. An outlier is identified by the oblique arrow above the unity line where $\Delta \mathrm{SUV}_{\text {peak }}>\Delta \mathrm{SUV}$ same. In plot (b), the tumour response on $y$-axis is plotted for all 26 tumours on $x$-axis. Thresholds of $\pm 30 \%$ as defined by PERCIST were applied to separate individual tumours to different categories using either $\Delta \mathrm{SUV}_{\text {peak }}$ (red) or $\Delta \mathrm{SUV}_{\text {same }}$ (blue). $19 \%$ of the tumours (5 out of 26) were ambiguously classified as shown by vertical arrows.

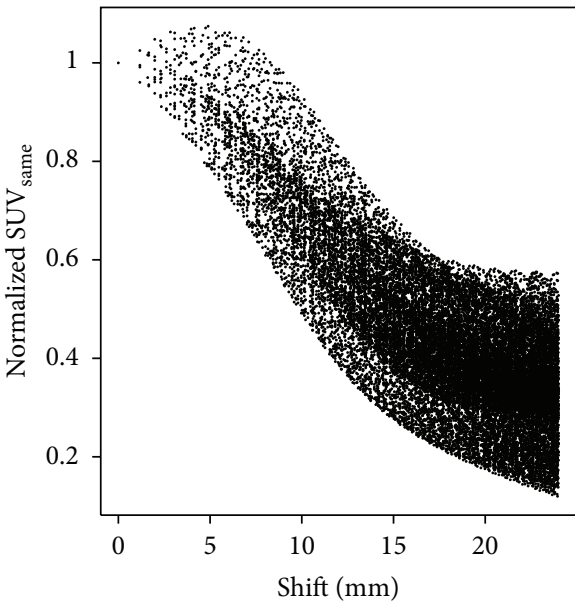

(a)

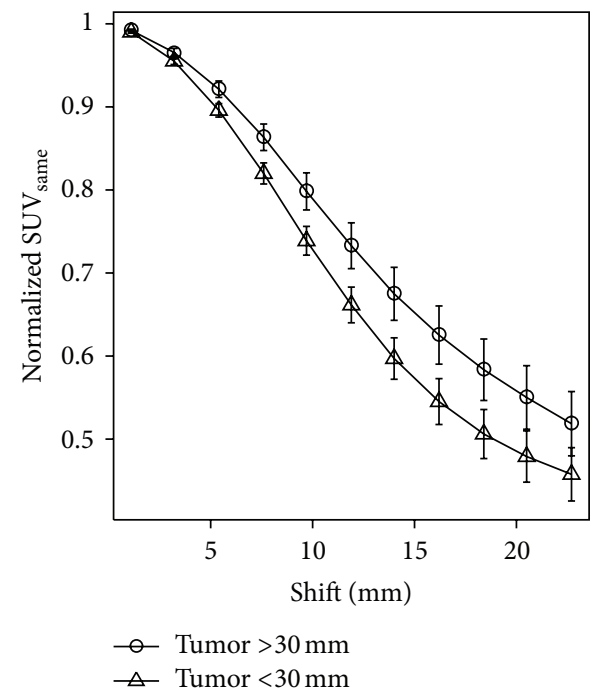

(b)

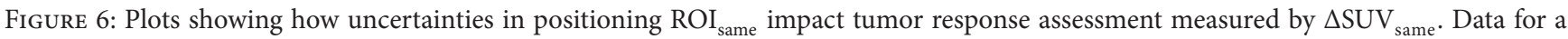
sample tumor (a) and the average data for all tumors (b) are shown. The error bars represent standard errors.

The PERCIST thresholds of $\pm 30 \%$ were applied to classify individual tumours into three categories of partial response, stable disease, and progressive disease. In 19\% (5 out of 26 ) of the tumours this resulted in ambiguous tumour classification depending on the ROI method as shown by the arrows.

Figure 6(a) shows an example plot for a tumour, demonstrating how uncertainties in positioning $\mathrm{ROI}_{\text {same }}$ may impact
$\Delta \mathrm{SUV}_{\text {same }}$ values. This plot shows that positioning the $\mathrm{ROI}_{\text {same }}$ a few millimeters away may decrease or increase $\Delta \mathrm{SUV}_{\text {same }}$ depending on whether $\mathrm{ROI}_{\text {same }}$ is moving towards the maximum uptake point or is moving away from it. However, by moving a few centimeters away, the points eventually start to drop since the $\mathrm{ROI}_{\text {same }}$ is sampling the background normal tissue uptake. Individual plots such as 


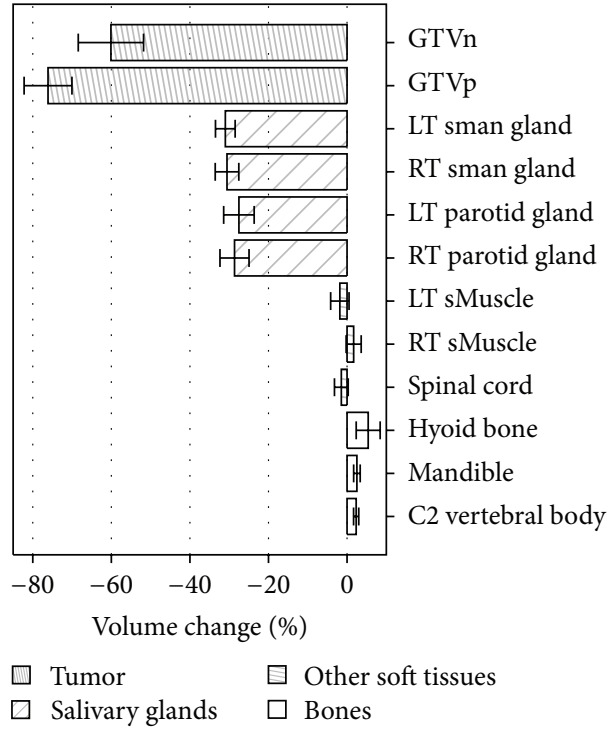

(a)

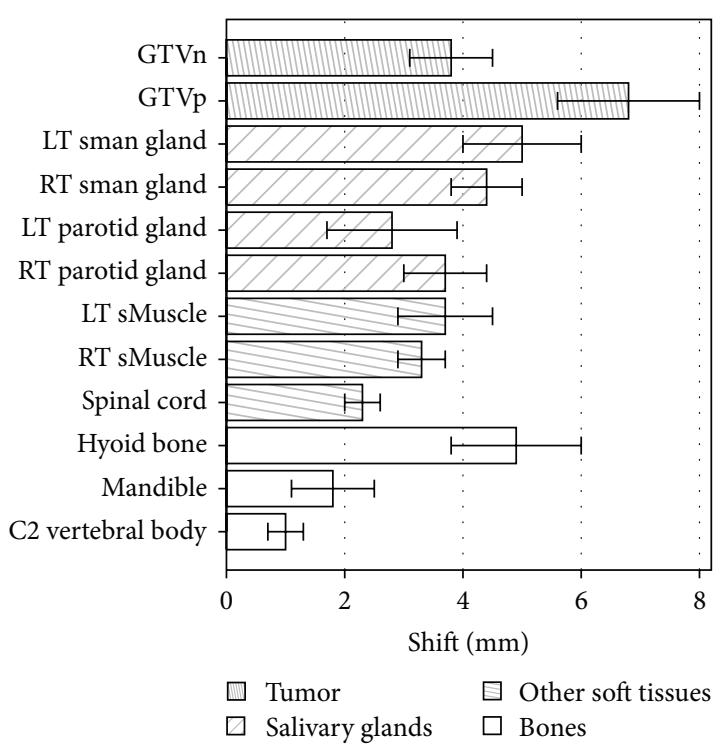

(b)

FIGURE 7: Geometric changes due to therapy for GTV and normal tissues characterized by percentage volume changes (a) and shifts (b). The bars show the median values and the error bars show the standard errors. GTVp: gross tumor volume (primary), GTVn: involved lymph node, LT: left, RT: right, sman: submandibular, sMuscle: sternocleidomastoid muscle.

Figure 6(a) were averaged for all GTVs. The results are shown as two curves in Figure 6(b) based on Intra-Tx GTV size bigger or smaller than $30 \mathrm{~mm}$. A statistically significant difference between the two curves at the $95 \%$ confidence level was found to be between $5.4 \mathrm{~mm}$ and $16.2 \mathrm{~mm}$.

3.2. Population B. A total of 97 normal tissue regions were contoured in 10 patients in both Pre-Tx and Intra-Tx. Figure 7 shows geometric changes due to therapy characterized in terms of percentage volume changes and COM shifts. Figure 7(a) shows the percentage volume changes for all GTVs and normal tissues. Negative volume changes indicate a loss of volume during therapy. Both GTVp and GTVn showed significant volume losses with median values of $76.1 \%$ and $60.1 \%$, respectively. The median volume loss for all GTVs was $67.2 \%$ (range, $8.4-96.9 \%$ ).

For normal tissues, significant volume losses were only found for the salivary glands. Median volume losses were 28.1\% (range, $7.3-45.6 \%$ ) for all parotid glands and $31.0 \%$ (range, 13.3-48.7\%) for all submandibular glands. Other soft tissues (i.e., sternocleidomastoid muscles and spinal cord) and bones did not show significant volume losses.

Figure 7(b) shows COM shifts in GTVs and normal tissues. The median shift for all GTVs was $5.9 \mathrm{~mm}$ and the 95\% CI range was $4.4-7.6 \mathrm{~mm}$. The C2 vertebral body showed the smallest shift with a median of $1.0 \mathrm{~mm}$. Right and Left parotid glands showed median shift values of $3.7 \mathrm{~mm}$ and $2.8 \mathrm{~mm}$, respectively. The median shifts in medial directions for right and left parotid glands were $1.4 \mathrm{~mm}$ and $2.5 \mathrm{~mm}$, respectively.

\section{Discussion}

Geometric changes during therapy can be expected to influence the accuracy with which an expert can place the tumour
ROI and thus could affect tumour response assessment using the $\mathrm{ROI}_{\text {same }}$ approach. Our results in Figure 7 are similar to those reported earlier $[22,23]$. We found that the $95 \%$ CI for GTV COM shift is between 4.4 and $7.6 \mathrm{~mm}$. This range may represent the upper range of uncertainty in placing the Intra-Tx tumour ROI at the same location as the PreTx tumour ROI. However in practice, attempts are made to correct for the geometric changes to some extent using anatomical landmarks. Moreover, in our study, population A had earlier Intra-Tx scans than population B. Due to these two factors, we expect that the uncertainties in placing the Intra-Tx tumour ROI have a smaller range than the $95 \% \mathrm{CI}$ shift, possibly in the $0-5 \mathrm{~mm}$ range. Based on Figure $6(\mathrm{~b})$, the impact of this uncertainty can be expected to be less than $10 \%$ on the measure of tumour response.

The placement of the fixed size ROI could have a significant effect on PET quantification for tumour response assessment. In this study, we found that $\Delta S U V_{\text {peak }}$ was $7.9 \%$ lower than $\triangle S U_{\text {same }}$ on average, and difference was up to $36 \%$. This degree of difference leads to different response assessment using PERCIST [16], resulting in overall $19 \%$ (5 out of 26) ambiguous tumour response assessment (Figure 5(b)). This finding underscores the need for an optimized PET quantification method in individual patients using a consistent and standard ROI for an accurate response assessment. A small fixed size ROI placed on a single slice is a simplistic approach to sample tumour uptake. Figure 2 demonstrates that the change in heterogeneity within the tumour due to treatment could be significant. This indicates the disadvantage of PET quantification for response assessment using a small fixed size ROI [24].

With $\triangle \mathrm{SUV}_{\text {peak }}$ one may risk overestimating response to treatment compared to $\triangle S_{U V} V_{\text {same }}$. This difference directly results from the fact that $\Delta \mathrm{SUV}_{\text {peak }}$ was on average $13.4 \%$ 
higher than $\mathrm{SUV}_{\text {same }}$ since it was centered at the maximum uptake point. Occasionally, $\mathrm{SUV}_{\text {peak }}$ may be smaller than $\mathrm{SUV}_{\text {same }}$. The outlier in Figure 5(a) corresponds to a situation where the central pixel of $\mathrm{ROI}_{\text {peak }}$ has a high uptake but its surrounding pixels have a lower uptake than the pixels within $\mathrm{ROI}_{\text {same }}$. Noisy PET images or high intratumour uptake heterogeneity might cause such a situation.

Considering the typical response thresholds which have been used to separate responding patients from nonresponding patients (last column in Table 1), the difference of $7.9 \%$ (and up to 36\%) between the two ROI methods could be clinically significant.

Many recent studies on early tumour response assessment have used the single-voxel based SUV $V_{\max }$ method, while the new recommendation favors a fixed size ROI as a more robust alternative to reduce uncertainties due to noise [16]. The placement of the fixed size ROI in Intra-Tx, whether $\mathrm{ROI}_{\text {peak }}$ or ROI $\mathrm{Rame}_{\text {sas }}$ as EORTC recommendations [20], could lead to significant uncertainties in response assessment. Thus, more studies are required to determine if either of these simple, fixed size ROI approaches are useful in assessing treatment response.

We found that the two ROI methods gave rise to highly correlated $(r=0.93)$ response assessments (Figure 5(a)). This high correlation is a direct result of high correlation $(r=0.92)$ between the SUV values of the two Intra-Tx ROI methods. This suggests that the higher uptake in $\mathrm{ROI}_{\text {peak }}$ also means potentially higher uptake in $\mathrm{ROI}_{\text {same }}$. $\mathrm{ROI}_{\text {same }}$ in general was sampling a different part of the tumour at some distance away

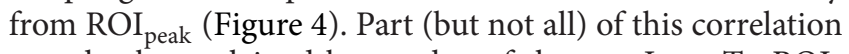
can also be explained by overlap of the two Intra-Tx ROIs (both $15 \mathrm{~mm}$ in diameter). In our patients, the two ROIs were in the same slice in only $8 \%$ of the GTVs. It is unsurprising that the pattern of tumour uptake could be considerably changed in response to therapy. Even without therapy, the pattern of uptake over time may alter as the tumour grows.

The EORTC [20] recommends placing the Intra-Tx ROI at the same anatomical location as the Pre-Tx ROI in order to sample the same area. This is a reasonable approach, that is, to evaluate the same location before and after some therapeutic intervention. It does not seem as intuitively reasonable to use the $\mathrm{ROI}_{\text {peak }}$ approach, which could mean comparing two anatomically distinct parts of the tumour before and after therapy. However, in a limited number of patients, we found that the two ROI methods were highly correlated $(r=0.93)$. This suggests that the two response assessment methods would likely have a similar accuracy in terms of differentiating responders versus nonresponders, although with different optimal response threshold values. In order to determine if a simple fixed ROI-based method has true utility for assessing response, substantial clinical trial data including patient outcomes is required. Such trial data could also be used to establish if there are threshold levels for ROI-based techniques that could reliably separate responders from nonresponders for each disease site and given treatment type.

\section{Conclusion}

PET quantification for assessing treatment response using a fixed size ROI is sensitive to the placement of the ROI within the tumour. The difference between the current recommendations favoring $\mathrm{ROI}_{\text {peak }}$ (over $\mathrm{ROI}_{\max }$ ) and earlier recommendations using $\mathrm{ROI}_{\text {same }}$ could be substantial (36\%) resulting in ambiguous treatment response assessment (19\%). Methods making use of such small ROIs have the advantage of being relatively simple to implement while still providing improved statistical properties versus the $\mathrm{SUV}_{\max }$ single voxel method. However, simplicity is not always an advantage and the use of a small fixed size ROI for tumour response assessment should be approached with caution in heterogeneous tumours. Clinical trials are necessary to compare the efficacy of a fixed size ROI over $\mathrm{ROI}_{\max }$ and establish a reliable threshold in a given cancer site.

\section{Acknowledgments}

This work was supported in part by the Ontario Institute for Cancer Research, a Beatty Fellowship, and an Ontario Graduate Scholarship. The authors thank Dr. Daniel Mozeg and Ms. Maggie Kusano for their scientific contributions. No conflict of interests exists.

\section{References}

[1] M. Schelling, N. Avril, J. Nährig et al., "Positron emission tomography using [(18)F]fluorodeoxyglucose for monitoring primary chemotherapy in breast cancer," Journal of Clinical Oncology, vol. 18, no. 8, pp. 1689-1695, 2000.

[2] W. A. Weber, V. Petersen, B. Schmidt et al., "Positron emission tomography in non-small-cell lung cancer: prediction of response to chemotherapy by quantitative assessment of glucose use," Journal of Clinical Oncology, vol. 21, no. 14, pp. 2651-2657, 2003.

[3] N. Avril, S. Sassen, B. Schmalfeldt et al., "Prediction of response to neoadjuvant chemotherapy by sequential F-18fluorodeoxyglucose positron emission tomography in patients with advanced-stage ovarian cancer," Journal of Clinical Oncology, vol. 23, no. 30, pp. 7445-7453, 2005.

[4] E. Brun, E. Kjellén, J. Tennvall et al., "FDG pet studies during treatment: prediction of therapy outcome in head and neck squamous cell carcinoma," Head and Neck, vol. 24, no. 2, pp. 127-135, 2002.

[5] C. Rousseau, A. Devillers, C. Sagan et al., "Monitoring of early response to neoadjuvant chemotherapy in stage II and III breast cancer by [18F]fluorodeoxyglucose positron emission tomography," Journal of Clinical Oncology, vol. 24, no. 34, pp. 5366-5372, 2006.

[6] J. A. Maisonobe, C. A. Garcia, H. Necib et al., "Comparison of PET metabolic indices for the early assessment of tumour response in metastatic colorectal cancer patients treated by polychemotherapy," The European Journal of Nuclear Medicine and Molecular Imaging, vol. 40, no. 2, pp. 166-174, 2013.

[7] K. Ott, W. A. Weber, F. Lordick et al., "Metabolic imaging predicts response, survival, and recurrence in adenocarcinomas of the esophagogastric junction," Journal of Clinical Oncology, vol. 24, no. 29, pp. 4692-4698, 2006. 
[8] W. A. Weber, K. Ott, K. Becker et al., "Prediction of response to preoperative chemotherapy in adenocarcinomas of the esophagogastric junction by metabolic imaging," Journal of Clinical Oncology, vol. 19, no. 12, pp. 3058-3065, 2001.

[9] H. A. Wieder, K. Ott, F. Lordick et al., "Prediction of tumor response by FDG-PET: comparison of the accuracy of single and sequential studies in patients with adenocarcinomas of the esophagogastric junction," The European Journal of Nuclear Medicine and Molecular Imaging, vol. 34, no. 12, pp. 1925-1932, 2007.

[10] K. Ott, U. Fink, K. Becker et al., "Prediction of response to preoperative chemotherapy in gastric carcinoma by metabolic imaging: results of a prospective trial," Journal of Clinical Oncology, vol. 21, no. 24, pp. 4604-4610, 2003.

[11] H. A. Wieder, B. L. D. M. Brücher, F. Zimmermann et al., "Time course of tumor metabolic activity during chemoradiotherapy of esophageal squamous cell carcinoma and response to treatment," Journal of Clinical Oncology, vol. 22, no. 5, pp. 900-908, 2004.

[12] J. D. Schwarz, M. Bader, L. Jenicke, G. Hemminger, F. Jänicke, and N. Avril, "Early prediction of response to chemotherapy in metastatic breast cancer using sequential 18F-FDG PET," Journal of Nuclear Medicine, vol. 46, no. 7, pp. 1144-1150, 2005.

[13] S. Ben-Haim and P. Ell, "18F-FDG PET and PET/CT in the evaluation of cancer treatment response," Journal of Nuclear Medicine, vol. 50, no. 1, pp. 88-99, 2009.

[14] M. E. Juweid and B. D. Cheson, "Positron-emission tomography and assessment of cancer therapy," The New England Journal of Medicine, vol. 354, no. 5, pp. 496-507, 2006.

[15] W. A. Weber and R. Figlin, "Monitoring cancer treatment with PET/CT: does it make a difference?" Journal of Nuclear Medicine, vol. 48, supplement 1, pp. 36S-44S, 2007.

[16] R. L. Wahl, H. Jacene, Y. Kasamon, and M. A. Lodge, "From RECIST to PERCIST: evolving considerations for PET response criteria in solid tumors," Journal of Nuclear Medicine, vol. 50, no. $1,2009$.

[17] T. Beyer, J. Czernin, and L. S. Freudenberg, "Variations in clinical PET/CT operations: results of an international survey of active PET/CT users," Journal of Nuclear Medicine, vol. 52, no. 2, pp. 303-310, 2011.

[18] R. Boellaard, N. C. Krak, O. S. Hoekstra, and A. A. Lammertsma, "Effects of noise, image resolution, and ROI definition on the accuracy of standard uptake values: a simulation study," Journal of Nuclear Medicine, vol. 45, no. 9, pp. 1519-1527, 2004.

[19] N. C. Krak, R. Boellaard, O. S. Hoekstra, J. W. R. Twisk, C. J. Hoekstra, and A. A. Lammertsma, "Effects of ROI definition and reconstruction method on quantitative outcome and applicability in a response monitoring trial," The European Journal of Nuclear Medicine and Molecular Imaging, vol. 32, no. 3, pp. 294-301, 2005.

[20] H. Young, R. Baum, U. Cremerius et al., "Measurement of clinical and subclinical tumour response using [18F]fluorodeoxyglucose and positron emission tomography: review and 1999 EORTC recommendations," The European Journal of Cancer, vol. 35, no. 13, pp. 1773-1782, 1999.

[21] M. van Herk and H. M. Kooy, "Automatic three-dimensional correlation of CT-CT, CT-MRI, and CT-SPECT using chamfer matching," Medical Physics, vol. 21, no. 7, pp. 1163-1178, 1994.

[22] J. L. Barker Jr., A. S. Garden, K. K. Ang et al., "Quantification of volumetric and geometric changes occurring during fractionated radiotherapy for head-and-neck cancer using an integrated CT/linear accelerator system," International Journal of Radiation Oncology Biology Physics, vol. 59, no. 4, pp. 960970, 2004.

[23] C. Lee, K. M. Langen, W. Lu et al., "Evaluation of geometric changes of parotid glands during head and neck cancer radiotherapy using daily MVCT and automatic deformable registration," Radiotherapy and Oncology, vol. 89, no. 1, pp. 8188, 2008.

[24] M. Vanderhoek, S. B. Perlman, and R. Jeraj, "Impact of the definition of peak standardized uptake value on quantification of treatment response," Journal of Nuclear Medicine, vol. 53, no. 1, pp. 4-11, 2012. 


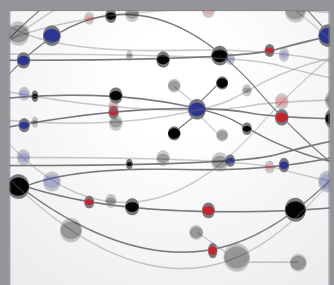

The Scientific World Journal
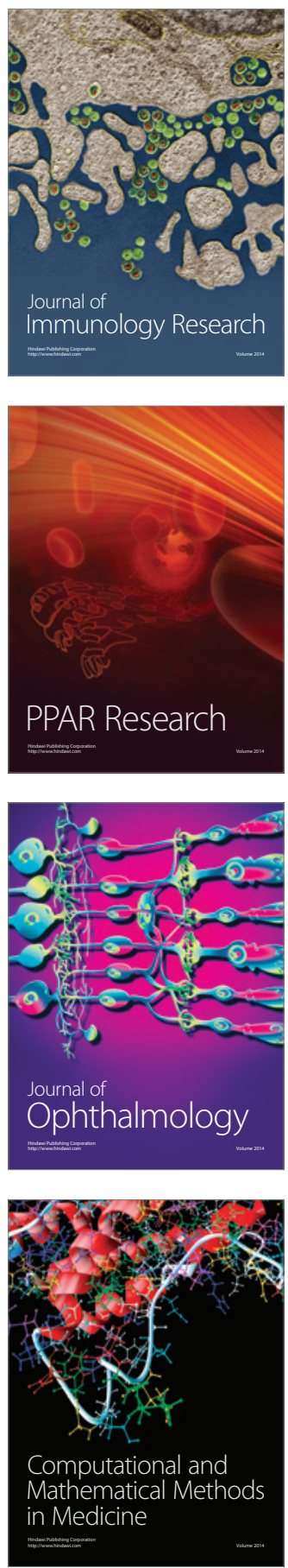

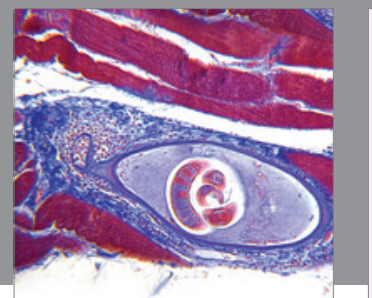

Gastroenterology

Research and Practice
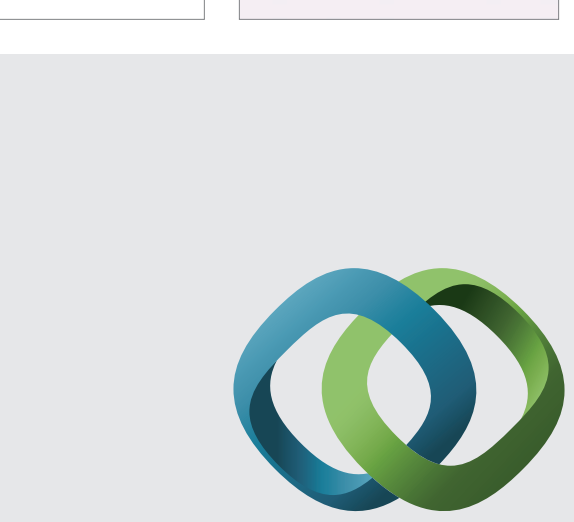

\section{Hindawi}

Submit your manuscripts at

http://www.hindawi.com
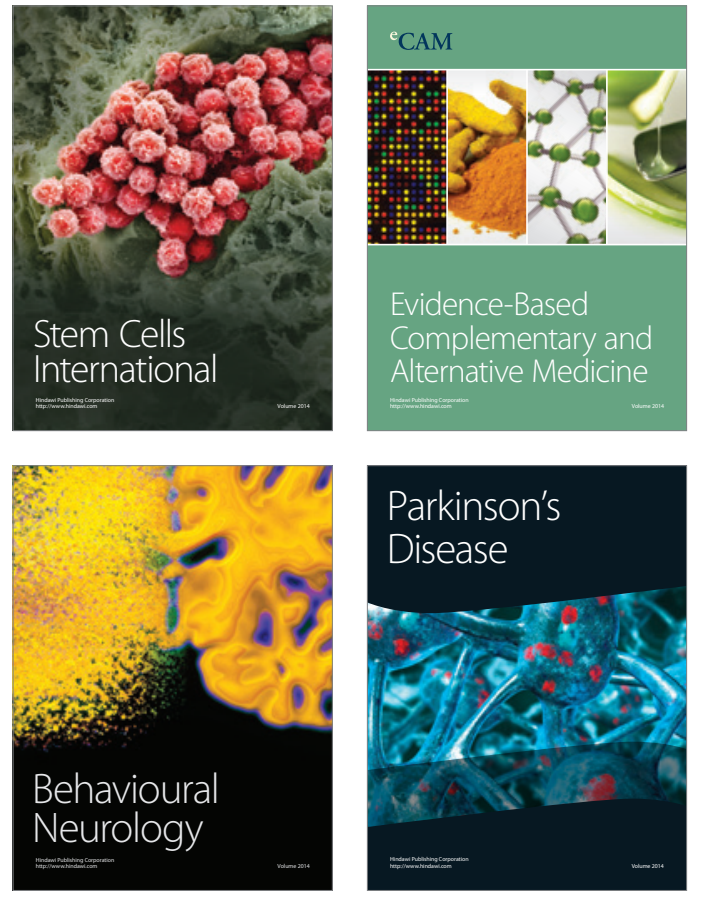
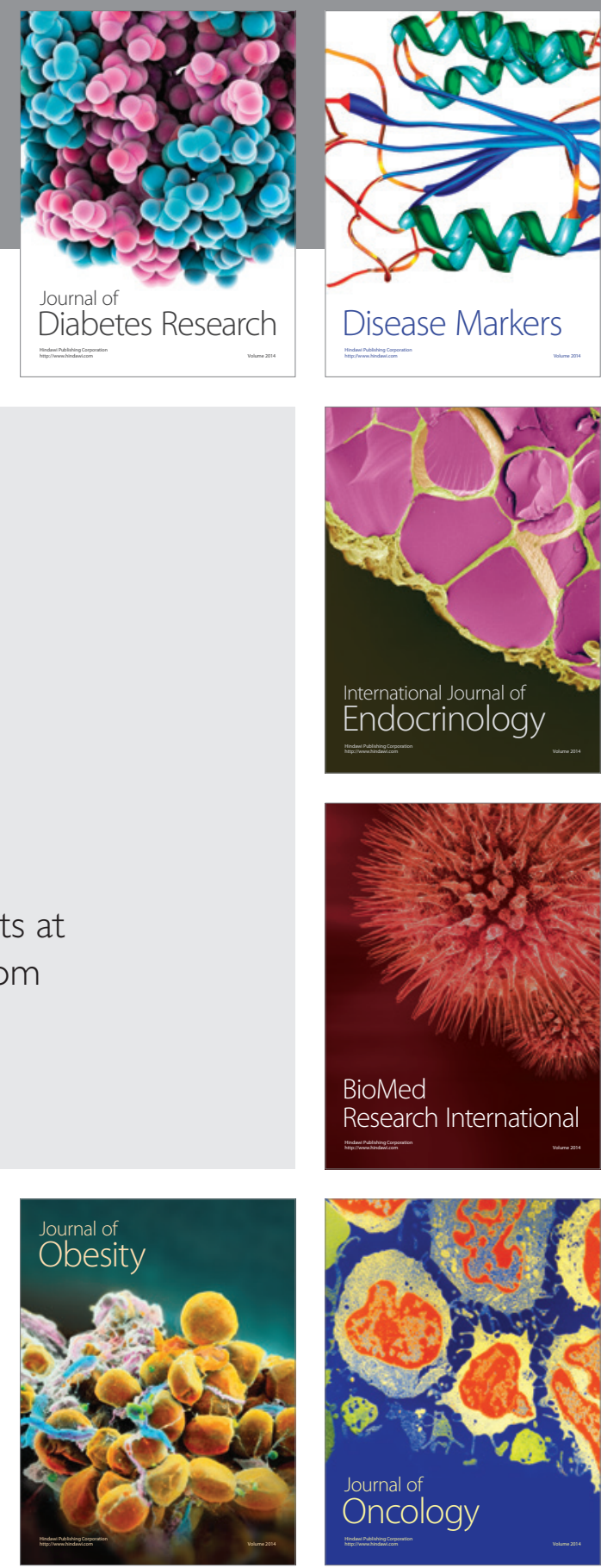

Disease Markers
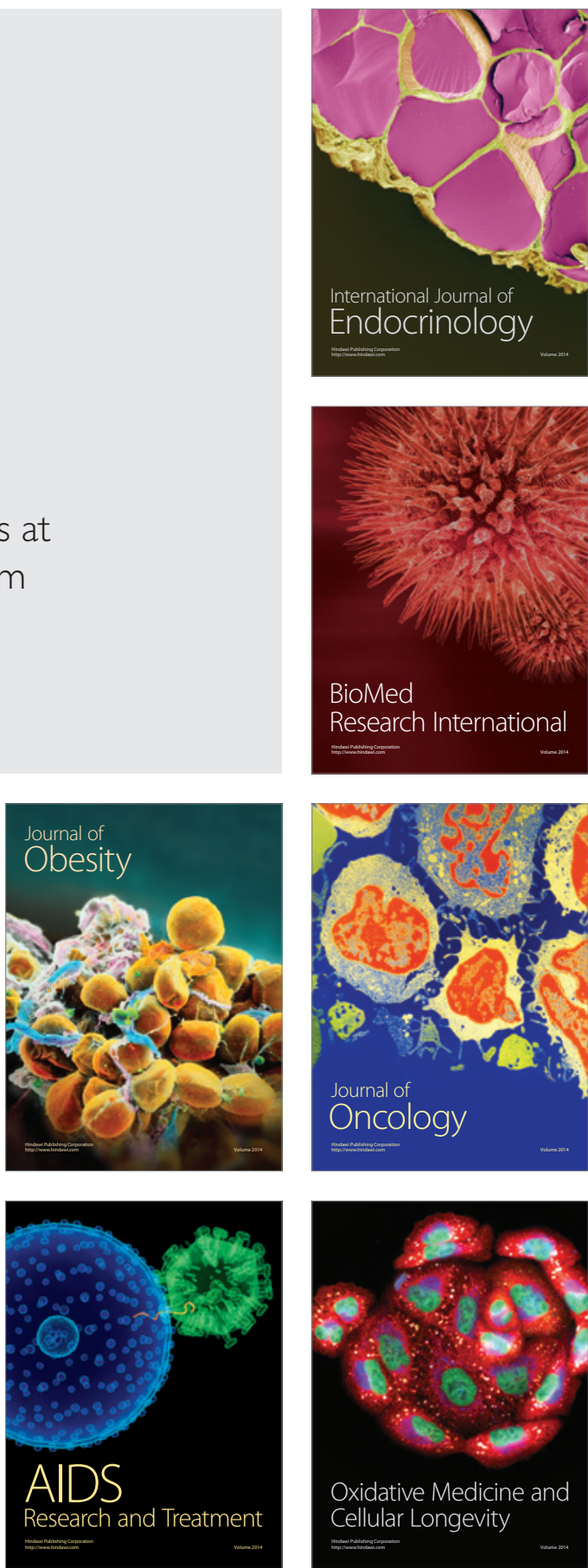